\title{
Contention-Based Geographic Forwarding Strategies for Wireless Sensors Networks
}

\author{
Carlos H. M. de Lima, Member, IEEE, Pedro H. J. Nardelli, Member, IEEE, Hirley Alves, Member, IEEE, \\ and Matti Latva-aho, Senior Member, IEEE
}

\begin{abstract}
This paper investigates solutions that combine relay selection algorithms and opportunistic geographic routing strategies for autonomous wireless sensor networks, wherein transmissions often occur over multiple hops. In order to design and assess such contention-based geographic forwarding strategies, we establish an analytical framework that incorporates concepts from probability generating functions (through power series expansion), spatial point processes, and auction theory, as well as Monte Carlo simulations to corroborate analytical results. The investigated strategies are built upon three constituent parts: 1) relay selection algorithm; 2) contention resolution mechanism; and 3) geographic forwarding strategy. We then propose an auction-based algorithm for selecting the relay node that relies on the network topology as side-information. Our results show that geographic solutions that iteratively exploit the local knowledge of the topology to ponder the algorithm operation outperforms established random approaches. Although, there is an intrinsic tradeoff between the time needed to identify a suitable next hop relay and the respective progress that is achieved toward the final destination.
\end{abstract}

Index Terms-Random processes, relay networks, routing protocols, ad hoc networks, transforms, auction theory.

\section{INTRODUCTION}

$\mathbf{L}$ ATELY, wireless communications are becoming a key technology for autonomous networks by providing means to deploy a wide range of emerging applications, such as smart houses, smart factories and networked cars. Wireless sensor networks are attractive for many reasons: low implementation and maintenance costs, flexible (physical) topology, as well as scalability [1]. Additionally, it enable information exchange between autonomous devices without any (direct) human intervention [1]-[4]. However, a major challenge for wireless sensor networks is to cope with the inherent requirements of such application. For instance, in an industrial environment these requirements are often more stringent than home environments due to the presence of highly reflective materials and additional interference from the machinery, which limits performance due to the increase interference profile [5]. Then,

Manuscript received October 7, 2015; revised December 14, 2015; accepted December 15, 2015. Date of publication December 17, 2015; date of current version February 10, 2016. This work was supported by the Academy of Finland, National Counsel of Technological and Scientific Development, and Special Visiting Researcher Fellowship Coordination for the Improvement of Higher Education Personnel under Grant 076/2012. The associate editor coordinating the review of this paper and approving it for publication was Prof. Okyay Kaynak.

C. H. M. de Lima is with São Paulo State University, São João da Boa Vista 13874-149, Brazil (e-mail: carlos.lima@sjbv.unesp.br).

P. H. J. Nardelli, H. Alves, and M. Latva-aho are with the Centre for Wireless Communications, University of Oulu, Oulu 90014, Finland (e-mail: nardelli@ee.oulu.fi; halves@ee.oulu.fi; matla@ee.oulu.fi).

Digital Object Identifier 10.1109/JSEN.2015.2509778 legacy point-to-point or point-to-multipoint protocols, which are well-established concepts in many wireless systems, does not seem to be suitable for industrial [5].

In this context, the use of short hops to form a wireless multi-hop link appear to be a simple, while efficient, solution for many applications from home to industrial environments. The literature of ad hoc networks provides several insights on how to analyze and build multi-hop systems. For instance, [6], [7] study the formation and maintenance of multi-hop connections in large-scale ad hoc networks. As a result of nodes' mobility, network dynamics and channel impairments, the wireless links undergo great fluctuation on their availability and quality. Specifically in [6], Baccelli et al. emphasize that opportunistic routing schemes, which dynamically form multi-hop links by selecting the most suitable relay at any slot and at any hop, outperform other routing mechanisms in such distributed scenarios. In fact, there are no fixed routers: any node in the network should be capable of relaying packets.

Additionally, as control and payload information share the same pool of available resources, the route management should avoid excessive overhead. Quality of Service (QoS) requirements must be also satisfied when optimizing the utilization of the network resources. When dealing with multi-hopping, the design decision of having a route over many short hops or over few long hops (i.e hopping strategy) is critical [7], [8]. However, regardless the strategy used, there still exists a negotiation period between the nodes to decide which is the most suitable relay.

Then, under such conditions, geographic routing appears as a viable solution since it captures the dynamic behavior of ad hoc networks [4] and reacts to topological changes on demand. Therefore, when addressing such distributed geographic routing strategies, a common approach assesses contention-based solutions in terms of the progress they provide towards the final destination. For instance in [9], the authors addressed the problem of defining the forwarding regions, determining their impact on the system performance. This provides clear guidelines for designing the geographic routing protocols. Then, [10] proposes a greed forwarding cluster-based algorithm which is resilient to topological variations due to network dynamics, which is shown to enhance system performance with low latency. Similarly, an initial assessment of contention-based relay selection strategies is performed in [11] and [12]. Additionally, a comprehensive review of hierarchical and geographic routing can be found, receptively, in [1] and [4]. 
This paper extends those results while focusing on identifying both the expected progress and the cost of finding a suitable relay at each hop. The analysis of the geographic routing strategies is conducted by assessing relay selection algorithms, contention resolution mechanisms, and geographic forwarding schemes. Specifically, the cost of selecting the next hop relay is characterized in terms of the distribution of the time necessary to resolve the contention, also known as the relay election process. The expected progress obtained at each hop is determined using analytic tools of stochastic geometry.

Then, we introduce two distinct contention-based schemes combining geographic forwarding designs and relay selection algorithms. Our first solution combines splitting tree algorithm (random relay selection) with sectoral decision regions, this allows us to define circular sectors in order to increase the probability of finding a relay, and thus reducing the contention resolution interval. On the other hand, our second solution resorts to an auction-based relay selection and convex-lenses decision region. In this solution, the decision area is halved at every round, which allows to eliminate from the auction some candidate relays, and therefore the contention resolution interval is significantly reduced.

Herein, we introduce an analytical framework rather than using a network level simulation-based evaluation as presented in [10]. Moreover, we extend the analysis provided in [10]-[12]. Our analytical framework is employed to first characterize the contention resolution interval of the selection algorithms, and then compare the performance of these different solutions. Finally, we assess how the network performs in terms of the packet expected forward distance and the cost of finding a relay at each hop. Succinctly, the proposed contention-based geographic forwarding scheme differs from other strategies found in the literature (e.g. [4]) by introducing a Dutch auction mechanism which advantageously exploits local network topology (throughout geographic region designs) so as to resolve contentions and select suitable relays in dense infrastructureless ad hoc networks. Hence, our contributions are summarized as follows:

- we characterize the statistics of the contention resolution interval length for the contention-based selection algorithms;

- we introduce a framework to assess the achievable progress attained by different geographic forwarding regions;

- we meld the aforesaid contributions into an analytical framework to jointly analyze the achievable progress and the negotiation overhead; and

- we design a new auction-based relay selection scheme for random multiple access networks (such as wireless sensor and ad-hoc networks) that recursively adapts the forwarding regions.

The reminder of this paper is organized as follows: Section II introduces the system model. In Section III-A describes the relay selection procedures, while Section III presents the geographic forwarding strategies. Next, Section IV provides an comprehensive set of numerical results and discussion. Finally, Section V concludes the paper.

\section{Problem Description And System Model}

\section{A. Problem Description}

Wireless sensor networks are highly dynamic systems composed of hundreds of communicating nodes. Therein, information needs to be continuously gathered and distributed so that routes to convey this traffic are established among such nodes [1]-[4]. Traditionally, pre-determined (quasi) static routes need to be discovered and maintained so as to enable inter node communication. In this context, geographic routing protocols emerged as a viable alternative to dynamically (re-)establish routes based on limited knowledge of network topology [4]. Therefore, it becomes necessary to establish a framework to design and evaluate how distinct geographic based routing protocols perform under various network deployment configurations. Herein, we investigate the performance of such contention-based geographic forwarding strategies for sensor networks. To do that, we assess not only the geographic progress towards the final destination at each hop, but also the time (in slots) to select the most suitable relay at hop basis. Stochastic geometry is used to model the network topology and capture the random disposition of nodes [13], [14].

\section{B. Network Deployment Model}

To construct our investigation scenarios, a finite number of transmitting nodes $N$ is uniformly scattered inside an arbitrary bounded region which is set as the network area and denoted by $\mathcal{W}$. Strictly speaking, we define a binomial point process $\Phi$ as the random pattern formed by the $N$ random points $\left\{\varphi_{1}, \varphi_{2}, \cdots, \varphi_{N}\right\}$ that are independent and identically distributed (i.i.d.) in the same compact set $\mathcal{W} \subset \mathbb{R}^{2}$ [14]-[17].

In this scenario, potential relays are randomly distributed over the network area following a binomial point process. Thereby, the distribution of the number of neighbors within source's transmission range corresponds to a general 2-dimensional binomial point process: the number of points within a given region is fixed while their positions are uniformly distributed [14, Definition 2]. Furthermore, communicating nodes use antennas with omni directional radiation pattern. For instance, Fig. 1 provides an example of such deployment. Notice that the pair of squared black dots represent source and destination, while the circles (black and white) depict the potential relays.

1) Channel Access: Nodes operate synchronously and packets are transmitted on a slot-by-slot basis. Nodes use the shared medium (air interface) by means of a contentionbased random multi-access scheme. A two state error-less channel model is used, where the air interface is either busy or idle. Assume a destructive interference amongst concurrent transmissions in which the only source of packet loss is collision. Every network node operates in half-duplex fashion, and therefore can both transmit and receive packets, though not simultaneously. In fact, a slotted-based medium access control so that nodes implement multiple access with collision avoidance through a Request to Send (RTS)/ Clear To Send (CTS) handshake mechanism [14]. As it was 
shown in [18], such contention resolution procedure enables using whatever node is available at any transmission time.

A simplified connectivity model based on the unit disk graph is employed [19]. In this random connectivity model, the Euclidean distances between nodes determine their connectivity. Thus, we assume a path-loss based model as in [8], since fading component is averaged out and becomes constant factors as detailed in [14] and [20]. Discs of equal diameter form a graph in which any two vertices are connected by an edge whenever one disc contains the center of the other. ${ }^{1}$ In this case, awake neighbors within the source's radio range are considered eligible relays - notice that nodes undergo a duty cycle so as to save battery. Yet nodes dwelling in the source's transmission range successfully receive packets and the only cause of errors are packet collisions. In what follows, it is assumed that source nodes are not affected by the hidden and exposed problems, since we are specifically addressing the iterations among potential relays.

Time-slotted collision-type channel with binary feedback (either collision or not) and gated channel access are employed. By the end of each transmission slot nodes are immediately and errorlessly aware of the feedback. The channel access algorithms specifies when packets may join the contention resolution transactions. New packets that appear during the resolution of the current conflict are buffered, i.e. the access to the channel is blocked to all that did not take part in the colliding slot originating the contention resolution interval that is afoot. In other words, any potential relay that wakes up during an ongoing resolution interval does not interfere on current transactions [21].

The contention-based geographic forwarding strategies are composed of two constituent parts: $(i)$ the contention-based relay selection mechanisms which identifies the most suitable (regarding the network topology) next hop relay to forward the message towards the final destination; and ( $i$ i) geographic forwarding strategies which establish the topological priorities to select suitable relays. In the following sections we details the above constituent parts: while the former is discussed in Section III-A, the latter is described in Section III.

\section{Contention-BASEd Geographic FORWARDING STRATEGIES}

When assessing position-centric network routing solutions [9], [22], the geographic forwarding strategies are a common topic in the literature and rely on geographic regions to iteratively identify the most suitable relays on a hop basis (selection mechanism iteration). We implement the contention-based geographic forwarding strategies by coupling two complementary parts: an intertwined contention resolution and relay selection algorithm which are detailed in Section III-A, as well as geographic forwarding decision regions which are presented in Section III. Succinctly, given a selection criterion, e.g. the geographical progress, the relay selection mechanism recursively searches for the most suitable

\footnotetext{
${ }^{1}$ Note that the fading component is averaged out and the long term path loss attenuation only is considered in the signal strength decay function so that the random connectivity model is distance based.
}

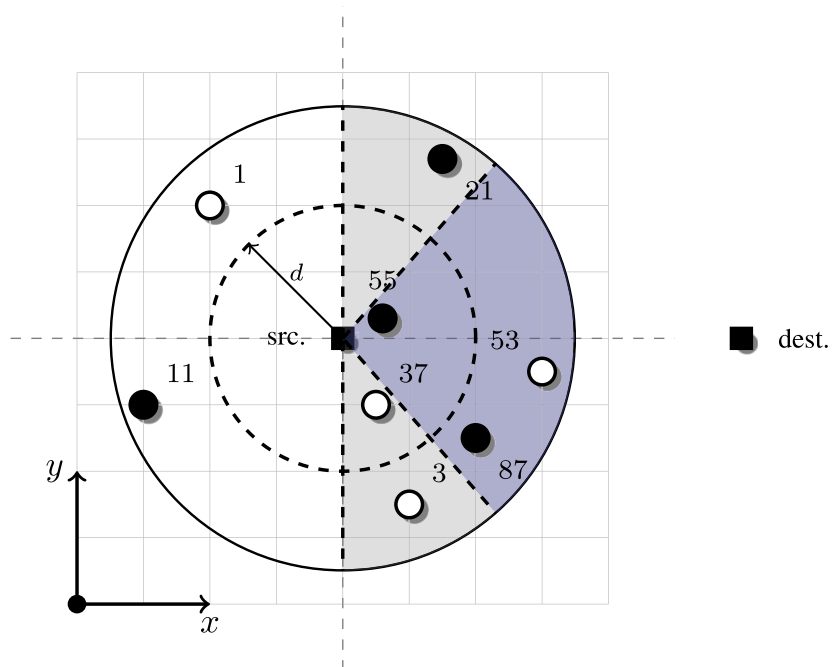

Fig. 1. Illustration of the sectoral decision region with $Q=2$ splitting groups and angular aperture of 180 degrees. Dashed lines defined the forwarding region (shaded regions in light gray and blue), black circles identify awake nodes and white circles identify asleep nodes. All awake neighbors within the shaded region are eligible relays.

relay through the levels of a binary search tree (branching process) by which the initial set of contending nodes are divided based on their priority to reply. In parallel, at each such conflict resolution round (tree branching) the respective decision regions are dynamically subdivided so as to separate subsets of potential relays based on their positions.

At each iteration of the relay selection mechanism, the decision regions determine the group of potential relays entitled to reply to the requesting source node at that specific transmission interval. The geographic forwarding strategies make use of the knowledge of the network topology, globally or locally, to route packets along multi-hop links. Relays are selected in a hop basis depending on their relative dispositions to the corresponding source: the node that provides the longest progress towards the destination is preferably chosen.

In this work, we consider two relay selection algorithms, namely the standard tree and auction-based algorithms. Moreover, we define two distinct decision regions: Sectoral Decision Region (SDR) and Convex Lenses Decision Region (CDR) as shown in Figs. 1 and 2, respectively. The former determines circular sectors with central angles which are adjusted at each iteration so as to halve the area of the decision region and match the probability of finding a suitable relay therein, while the latter establishes regions whose areas are halved at each round.

\section{A. Contention-Based Relay Selection Algorithm}

As mentioned earlier, two distinct contention-based relay selection mechanisms are analyzed: $(i)$ a totally random solution based solely on the standard splitting tree algorithm for performing random multiple access communications [23]; and (ii) an auction-based approach exploiting the local knowledge of network topology to avoid collisions and, whenever necessary, to shorten the contention resolution period [11]. 


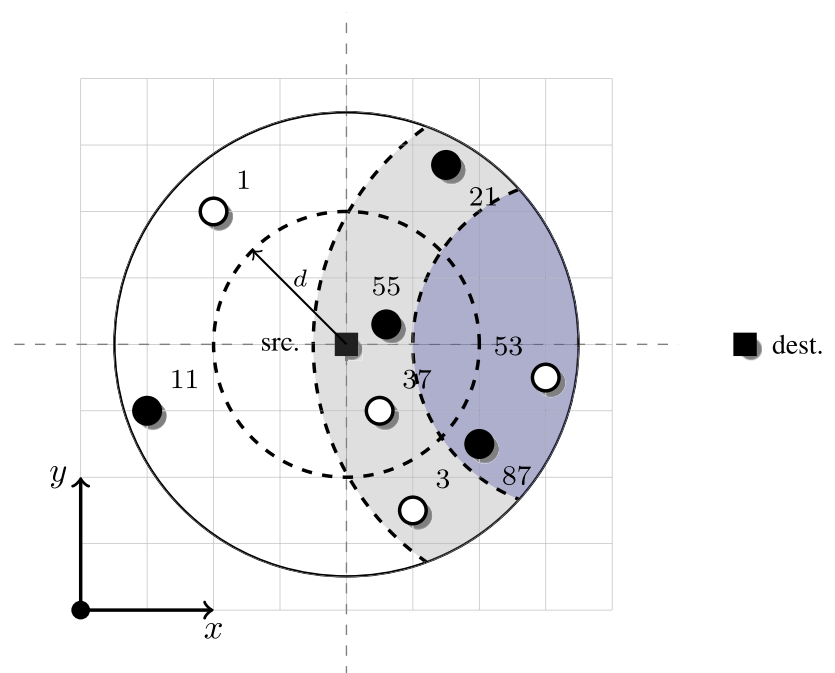

Fig. 2. Illustration of the second iteration of the convex lenses decision region with $Q=2$ regions. Dashed lines defined the forwarding region (shaded regions in light gray and blue), black circles identify awake nodes and white circles identify asleep nodes. All awake neighbors dwelling within the shaded region are eligible relays.

The contention resolution algorithms are classified in terms of the endured interval length, which is the necessary time to select the most suitable relay among all the eligible neighbors. It is worth to emphasize that only the time necessary to resolve the contentions is considered to analyze the negotiation cost in conflict resolutions algorithms. Neither the queuing time nor the (re)transmissions propagation time of the data payload are taken into consideration for the overhead assessment.

To characterize the cost of selecting a relay at each hop, Probability Generating Functions (PGFs) are used to represent the Probability Mass Function (PMF) of the Contention Resolution Interval (CRI) length by means of power series with non-negative coefficients. In other words, let $X$ be a discrete random variable taking non-negative integer values whose probability mass functions is given as $p(x)=\operatorname{Pr}\{X=x\}$ [24]. Then, according to [25] the PGF is a time series representation of the PMF given by,

$$
G(z)=\mathrm{E}\left[z^{x}\right]=\sum_{x=0}^{\infty} p(x) z^{x}
$$

allowing us to easily recover the probability distribution by taking higher order derivatives, which is given by [26]:

$$
\operatorname{Pr}\{X=x\}=\frac{G^{(x)}(0)}{x !}
$$

Nonetheless, such process in (2) may become intractable as pointed out in [27]. Therefore, herein we resort to a numerical approach whereby the probability mass function is recovered by inverting the corresponding probability generating function. This method is an approximation based on the Fourier series and is introduced by Abate and Whitt in [27]. Thus, the distribution of the length of the contention resolution interval, namely $L_{N}$, is approximated as:

$$
\widetilde{\operatorname{Pr}}\left\{L_{N}=k\right\}=\frac{1}{2 k r^{k}} \sum_{j=1}^{2 k}(-1)^{j} \Re\left[G_{N}\left(r e^{\frac{\pi j l}{k}}\right)\right],
$$

where $\Re(\cdot)$ returns the real part of the enclosed complex number, $l$ yields the imaginary unity, and $r$ is the radius of convergence.

1) PGF of the Splitting Tree Algorithm (STA)-Based Relay Selection Algorithm (RSA): The splitting tree algorithm constitutes the operational underpinning of the standard tree-based (random) solution, which is tailored to random multiple access communications [23]. The algorithm for contention resolution works with the channel access method, which in its turn dictates when new packets may join the transactions still in progress. A gated channel access algorithm, known as blocked access protocol, is used to control the ingress of new packets in the contention. Meaning that no new contending relay is allowed in the contention taking place once the resolution of the conflict has been already initiated.

According to this on-demand medium access control mechanism, the source node always initiates the relay selection transactions by issuing a RTS packet. The neighbors that listened to the source's requisition split themselves randomly and independently based on the common probability that dictates the likelihood of accessing the shared channel - totally random approach. If no suitable relay is found in a given relay selection interaction, the source node backs off. After a predefined interval, it restarts the relay selection procedure addressing (hopefully) new players.

Since the standard tree-based scheme is a random approach to select relays, the source node can only identify the most suitable relay after collecting the forwarding information from all the eligible relays. The performance of the such algorithm is addressed by means of computational simulations in [10]. Fig. 1 illustrates a snapshot of the sectoral decision region that is used in conjunction with the standard tree-based solution.

As aforesaid, the probability generating function of the contention resolution interval is determined in order to characterize the relay selection cost. Thus, we derive the probability generating function of $L_{N}$ that is the conditional contention resolution interval length when $N$ nodes initially collide. The conditional contention resolution interval length considering a $Q$-sided fair $\operatorname{coin}^{2}$ is: $L_{N}=1$ for $N \in\{0,1\}$, or $L_{N}=1+\sum_{j=1}^{Q} L_{I_{j}}$ when $N \geq 2$, where $L_{I_{j}}$ is the contention resolution interval length needed to resolve the contention in each subgroup $I_{j}$ such that $\sum_{j=1}^{Q} i_{j}=N$. Note that the computation of the statistics of the $L_{N}$ involves the summation of multiple random variables each of them corresponding to a particular splitting group (or even subsets $I_{j}$ thereof) as illustrated in Fig. 3.

For example, the probability mass function of the sum of only two independent discrete random variables $X$ and $Y$ is given by the convolution of their corresponding mass functions, i.e. $p_{X+Y}=p_{X} * p_{Y}$. One can see that computing the statistics of the $L_{N}$ (total CRI length) by convolving all

\footnotetext{
${ }^{2}$ Each side identifies a branch of the binary search tree.
} 


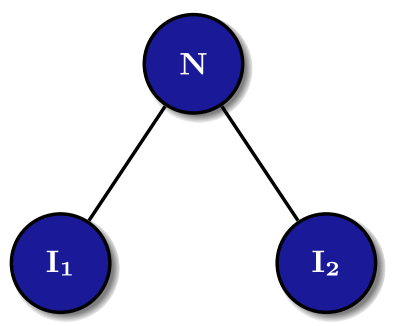

Fig. 3. Illustration of the binary search tree branching process.

subsets would be a laborious task. For that reason, probability generating functions are conveniently used herein so as to derive the distribution of the resolution interval length $L_{N}$. And for the standard tree-based approach is expressed as follows:

$$
G_{N}(z)=\sum_{k=0}^{\infty} \operatorname{Pr}\left\{L_{N}=k\right\} z^{k}=\mathrm{E}\left\{z^{L_{N}}\right\},
$$

where $L_{N}$ is a discrete random variable assuming nonnegative integer values representing the contention resolution intervals. Note that it follows from (1) and (4) we have $G_{0}(z)=G_{1}(z)=z$.

The expectation of (4) is then computed as

$$
\mathrm{E}\left\{z^{L_{N}}\right\}=\mathrm{E}\left\{z^{1+\sum_{j=1}^{Q} L_{I_{j}}} \mid N\right\},
$$

which can be rewritten as

$$
\begin{aligned}
\mathrm{E}\left\{z^{L_{N}}\right\}= & \sum_{i_{1}, \ldots, i_{Q}} \mathrm{E}\left\{z^{L} \mid I_{1}, \ldots, I_{Q}\right\} \\
& \times \operatorname{Pr}\left\{I_{1}=i_{1}, \ldots, I_{Q}=i_{Q}\right\},
\end{aligned}
$$

where

$$
\begin{aligned}
E\left\{z^{L_{N}}\right\} & =z E\left\{z^{L_{I_{1}}}\right\} \ldots E\left\{z^{L_{I_{Q}}}\right\} \\
& =z G_{L_{I_{1}}}(z) \ldots G_{L_{I_{Q}}}(z),
\end{aligned}
$$

and

$$
P\left\{I_{1}=i_{1}, \ldots, I_{Q}=i_{Q}\right\}=\left(\begin{array}{c}
N \\
i_{1}, \ldots, i_{Q}
\end{array}\right) \prod_{j=1}^{Q} P_{j}{ }^{i_{j}} .
$$

Finally, substituting (7) and (8) on (6), we obtain

$$
G_{N}(z)=z \sum_{i_{1}, \ldots, i_{Q}}^{N}\left(\begin{array}{c}
N \\
i_{1}, \ldots, i_{Q}
\end{array}\right) \prod_{j=1}^{Q} G_{L_{I_{j}}}(z) P_{j}{ }^{i_{j}} .
$$

where $P_{j} i_{j}$ is the probability of $i_{j}$ nodes flip the $j$ side of the $Q$-sided fair coin. Note that the summation in (6) iterates over all possible combinations of the multinomial splitting groups $i_{1}, \cdots, i_{Q}$.

Next the computation of the length of the contention resolution interval in (6) is particularized for the binary tree configuration alone (two splitting groups). Thus, $B_{N, i}=\left(\begin{array}{c}N \\ i\end{array}\right) p^{i}(1-p)^{N-i}$ yields the probability that exactly $i$ nodes toss 0 (first splitting group), and then transmit in the very next frame after the collision, where $p$ corresponds to the

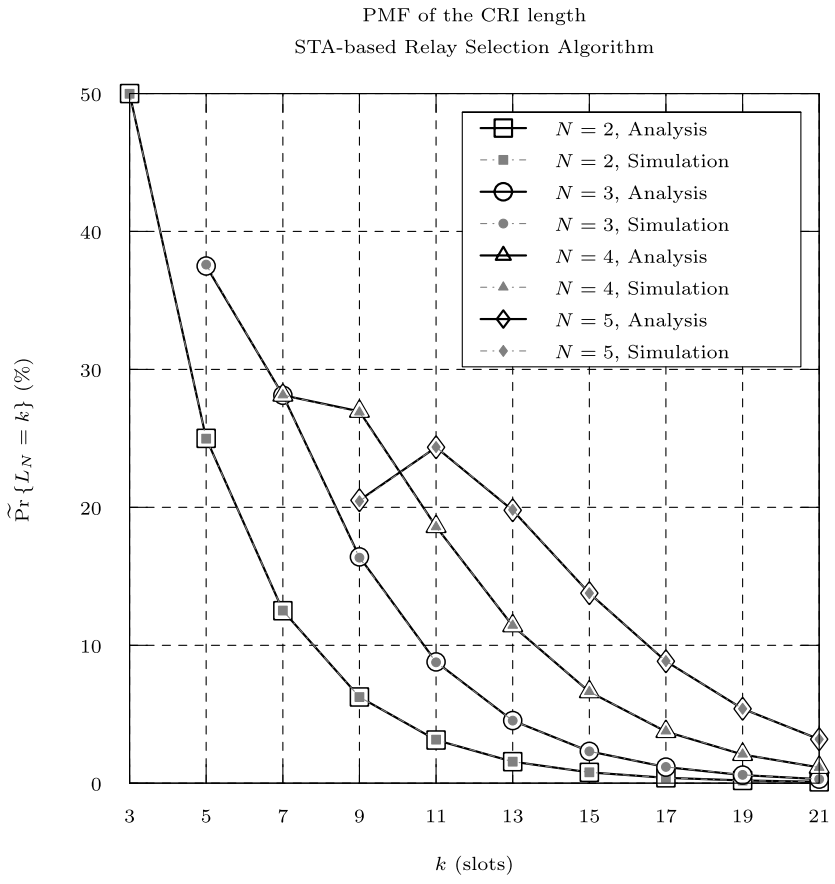

Fig. 4. PMF of the length of the contention resolution interval for the standard tree-based scheme. $N$ corresponds to the number of potential relays that initially collide. We consider $Q=2$ subgroups (two binary search tree branches).

probability of tossing 0 when using the unbiased binary coin for each choice. Finally, the probability generating function of the length for the standard tree-based relay selection scheme is then given by,

$$
G_{N}(z)=z \sum_{i=0}^{N} B_{N, i} G_{i}(z) G_{N-i}(z),
$$

where $G_{i}(z)$ addresses the collision among $i$ nodes that flipped 0 (1st subset), and $G_{N-i}(z)$ corresponds to the additional slots to resolve the collision among $N-i$ nodes that flipped 1 (2nd subset).

Fig. 4 presents the probability mass function of the contention resolution interval length when the random-based selection is employed. The number of nodes identify the number of candidate relays that initially collide. The distribution of the contention resolution interval length is generated for an increasing number of contending relays. When using the totally random approach, the contention resolution interval significantly lengthens with the initial number of colliding relays. In fact, the resolution of the conflict may linger too much time before the contention is resolved and the next-hop relay is elected. As a consequence, the achievable data rate per node is compromised.

It is worth noting that when randomly interacting through the relay election process, nodes may expose themselves to much higher channel contention and consequently squander the already limited radio resources. To reduce the conflict over the air interface, potential relays may ponder their participation in the election process beforehand by dynamically evaluating the characteristics affecting the transmission, such as local 
network topology, network dynamics and channel impairments.

2) PGF of the Auction-Based RSA: The auction-based relay selection algorithm iteratively exploits location information to solve conflicts and then shortens the resolution interval by pruning the binary conflict resolution tree. In [10], Dutch auctions ${ }^{3}$ are proposed as an effective alternative to address the relay selection process in conjunction with random multiple access methods, where the source is the "auctioneer" and potential relays are the "bidders". In fact, Authors in [29] showed that by shortening the contention resolution, the number of (re) transmissions and the corresponding energy consumption is significantly reduced. The price is then derived from the separation between source, relays and destination (similar to the simple greedy forwarding) [30]. In a similar way, authors use "asset selling problems" in [31] as a proper model to study relay selection for geographic forwarding in which $N$ offers arrive sequentially over discrete time slots, and the decision process ends with the seller choosing an offer (most appropriate relay).

Fig. 2 illustrates the computation of the forwarding regions regarding two splitting groups. In the first round of the auction, the candidate relays are divided into two groups, namely $\{\{87,21,55\},\{11\}\}$. Since nodes $\{87,21,55\}$ reply at the same time slot, a collision occurs. The source detects the collision in the first slot and recomputes the forwarding regions accordingly. Whenever the blocked access protocol is considered, the contending nodes can also recompute the forwarding regions independently by themselves. Node $\{11\}$ also detects the collision and, as nodes of higher priority have already replied, just drops out. Thereafter, relays in the first colliding area are reordered in the sequence $\{\{87\},\{21,55\}\}$. Finally, node $\{87\}$ replies and the auction finishes.

According to the auction-based relay selection algorithm, potential relays that have not replied in the previous slot but detected a collision drop out of the ongoing transaction. This intrinsic "tree pruning" means that whenever the first subset visited after a collision leads to another collision, the second subset is dropped. For $i>1$, instead of the contention resolution interval having full length $B_{N, i} G_{i}(z) G_{N-i}(z)$, the tree pruning procedure leads to a shorter length $B_{N, i} G_{i}(z)$ [32].

Note that the derivation of the probability generating function of the length of the contention resolution interval is conditioned on the size (number of contending nodes) of the initial set of colliding nodes. We can then express the probability generating function of the auction-based solution as follows:

$$
G_{N}(z)=z^{2} B_{N, 0} G_{N}(z)+z^{2} B_{N, 1}+z \sum_{i=2}^{N} B_{N, i} G_{i}(z),
$$

where the first term accounts for case when no reply is issued in the first slot, and the second term addresses the case when there is only one eligible node in the first decision region (first slot).

\footnotetext{
${ }^{3}$ Dutch auctions are extremely convenient to sell goods - assignment of network resources - quickly. The reasons are two-fold, the auction ends with the very first bid and the auctioneer may appropriately set the decreasing rate of the artifact value (depreciation rate) aiming at quickening the auction [28].
}

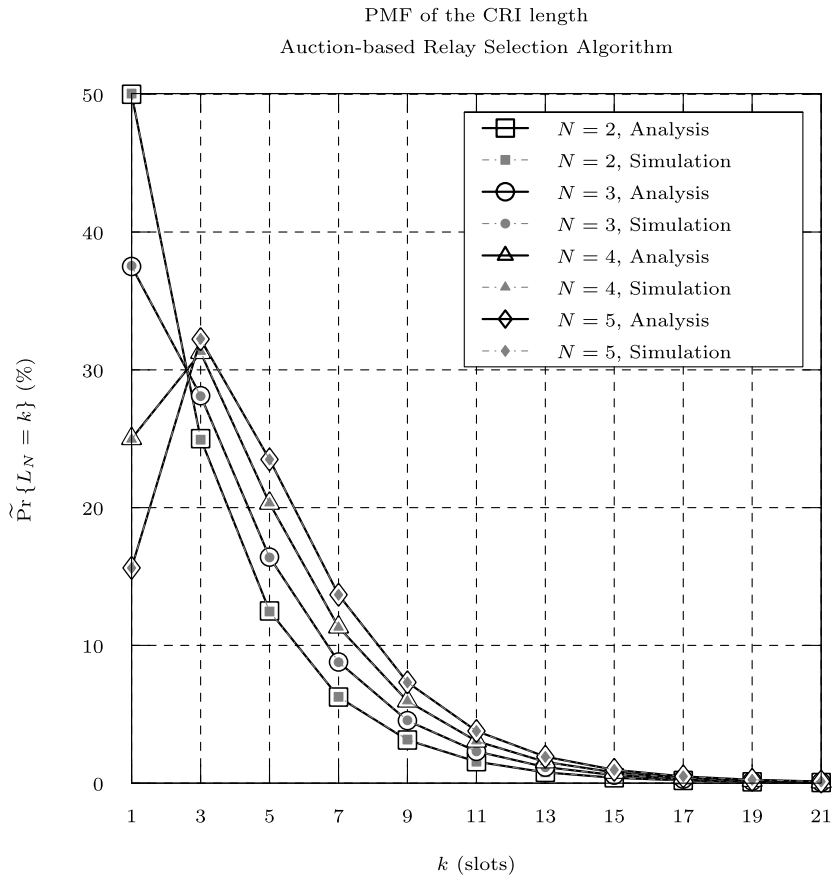

Fig. 5. PMF of the length of the contention resolution interval for the auction-based relaying selection. $N$ corresponds to the number of potential relays that initially collide. We consider $Q=2$ subgroups (two binary search tree branches).

Whenever nodes involved in a contention listen to an idle slot just after the colliding slot they do not need to undergo a collision in the subsequent slot. The auction-based solution can be refined in a way that nodes may split themselves into the decision regions prior to any indication of collision whereby the collision avoidance mechanism is characterized. This procedure leads to skipping one level of the binary tree, and is expressed by dropping one slot of the first term of (11), which then becomes

$$
G_{N}(z)=z B_{N, 0} G_{N}(z)+z^{2} B_{N, 1}+z \sum_{i=2}^{N} B_{N, i} G_{i}(z) .
$$

For the auction-based mechanism, the probability mass function of the length of the contention resolution interval is presented in Fig. 5. The impact of the initial number of colliding nodes on the duration of the resolution is still observed, though in much lesser extent. By using the location information, eligible nodes can independently split themselves into priority groups quickening the selection transactions in a distributed manner. The location awareness improves the contention resolution capability of the auction-based alternative, mainly when the initial number of colliding nodes are high.

From Figs. 4 and 5, regarding the curves of four eligible relays alone, the resolution probability for nine slots is nearly $28 \%$ for the random selection procedure, whereas the auctionbased one is approximately $8 \%$ only. If high resolution probability is allowed or large number of relays is available, the selection can occur much faster with auction-based algorithm.

\section{B. Geographic Decision Regions}

For the 2-dimensional binomial processes employed here, the complementary cumulative distribution function of the 
PDF of the distance to the nearest neighbor

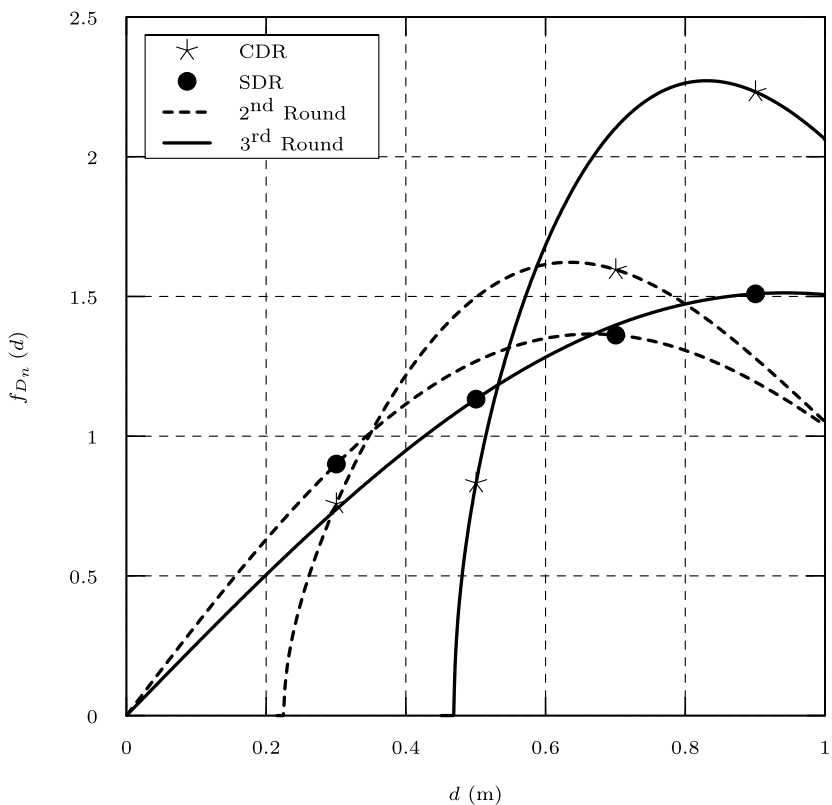

Fig. 6. Impact of the adaptation of the decision regions on the progress regarding the nearest eligible relay. The transmission range is $R=1 \mathrm{~m}$ and the number of candidate relays is $N=5$.

distance to the $n$th nearest neighbor $\bar{F}_{D_{n}}(d)$ is interpreted as the probability of existing less than $n$ points inside the decision region $\mathcal{B}[13] . \bar{F}_{D_{n}}(d)$ is well-known expression and is derived, for example, in [11]. Here, we only show the final expression due to space restrictions.

$$
\bar{F}_{D_{n}}(d)=\sum_{k=0}^{n-1}\left(\begin{array}{l}
N \\
k
\end{array}\right) p_{d}^{k}\left(1-p_{d}\right)^{N-k},
$$

from where Probability Density Function (PDF) $f_{D_{n}}(d)$ can be readily obtained.

Fig. 6 shows the results for the nearest eligible neighbor, while Fig. 7 addresses the furthest neighbor within source's radio range. Thus, after each collision, by considering partitions of the initial forwarding region that are closer to the final destination, the probability of finding nodes at further distances increases with each iteration, whereas the likelihood of having high number of contenders in smaller areas decreases. This is an important conclusion, because it impacts directly the contention resolution interval, since it more likely to find a relay and therefore reducing the length of the interval.

Figs. 8 and 9 illustrate the distribution of the distance to the $n$th nearest neighbor - obtained from (13) - when using the sectoral and convex-lenses decision region designs, respectively. In order to establish a fair comparison between the sectoral and convex lenses decision regions, we initially set the radius defining the forwarding region for the former (see Fig. 1) and latter (see Fig. 2) designs equal to the source node transmission range. Thereafter, we adjust the aperture angle defining the circular sector in Fig. 1 to have area equal to the corresponding lens in Fig. 2. Then, we have the same probability of finding nodes inside both decision regions.

As can be seen from Figs. 8 and 9, the obtainable results are fairly comparable in terms of the achievable advancements

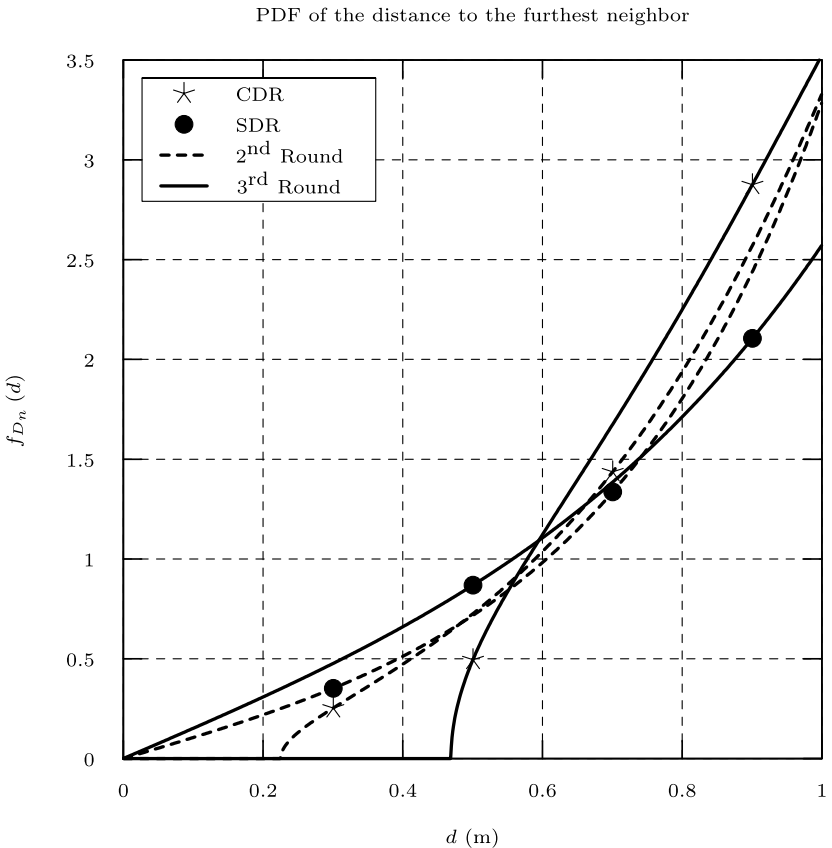

Fig. 7. Impact of the adaptation of the decision regions on the progress regarding the furthest eligible relay. The transmission range is $R=1 \mathrm{~m}$ and the number of candidate relays is $N=5$.

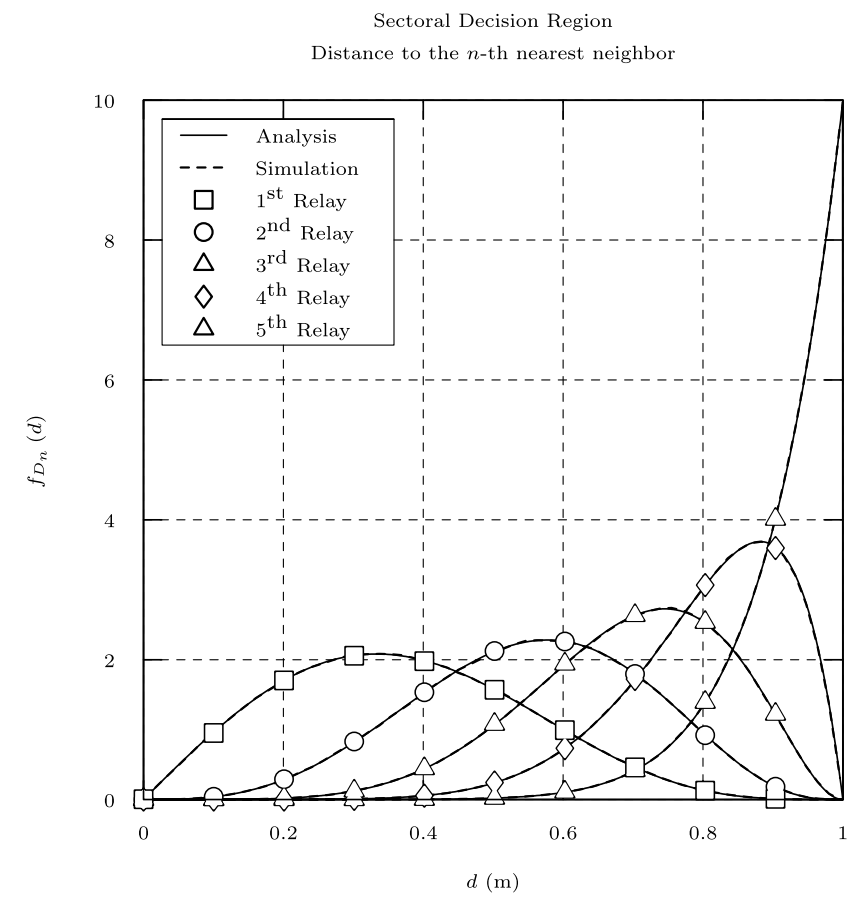

Fig. 8. PDF of the distance from a reference point to the $n$th neighbor when using the sectoral decision design. The transmission range is $R=1 \mathrm{~m}$ (normalized) and the number of candidate relays is $N=5$.

when there is an equivalence between the designs of the decision regions. On the other hand, when the effect of the recursive iterations of the auction-based scheme are taking into consideration on the re-computations of the forwarding regions after each collision, the benefits of adaptively resetting the convex-lenses regions become evident as shown in Figs. 6 and 7 above. 
Convex Lenses Decision Region

Distance to the $n$-th nearest neighbor

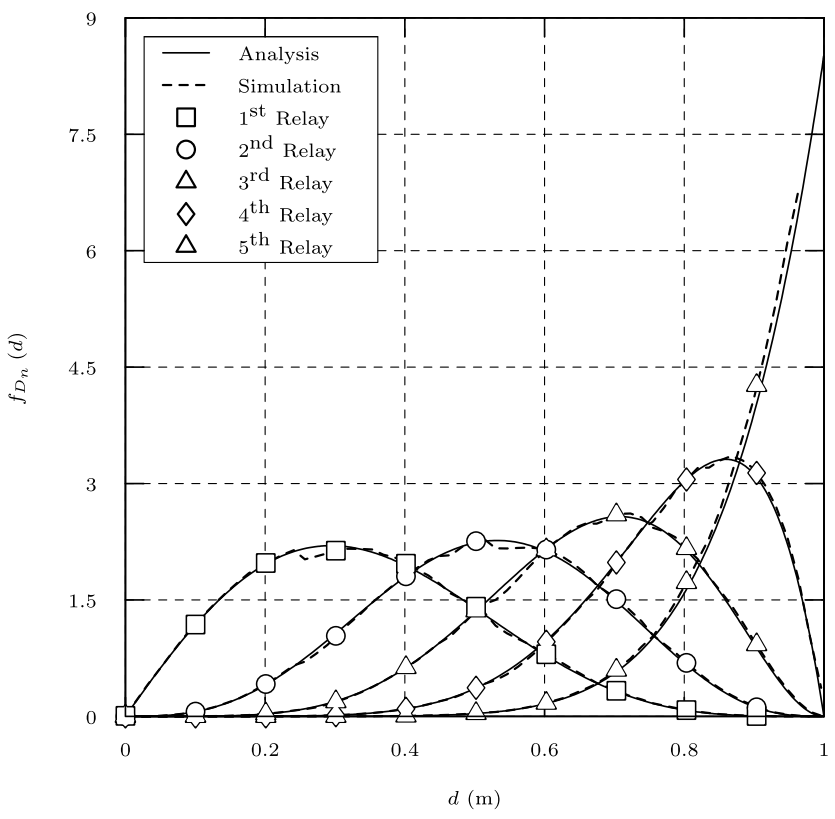

Fig. 9. PDF of the distance from a reference point to the $n$th nearest neighbor when using the convex-lenses design. The transmission range is $R=1 \mathrm{~m}$ (normalized) and the number of candidate relays is $N=5$.

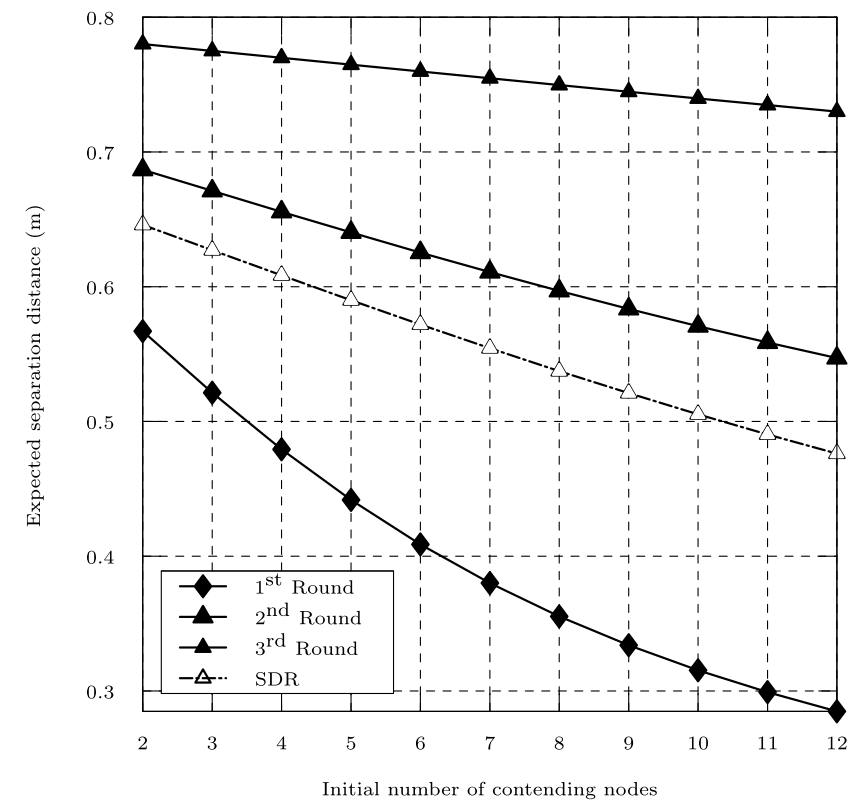

Fig. 10. Expected separation distance of the nearest neighbor for the proposed schemes. The source's transmission range is $R=1 \mathrm{~m}$ (normalized).

\section{RESULTS}

In this section, we use the analytical framework of Section III to assess the achievable progress towards the final destination and the corresponding negotiation overhead conditional on the initial number of colliding nodes. To do so, the achievable progress is weighed against the time to select a suitable relay so as to identify the most advantageous procedure. For the sectoral and the convex lenses decision regions, Figs. 10 and 11 show the expected value of the

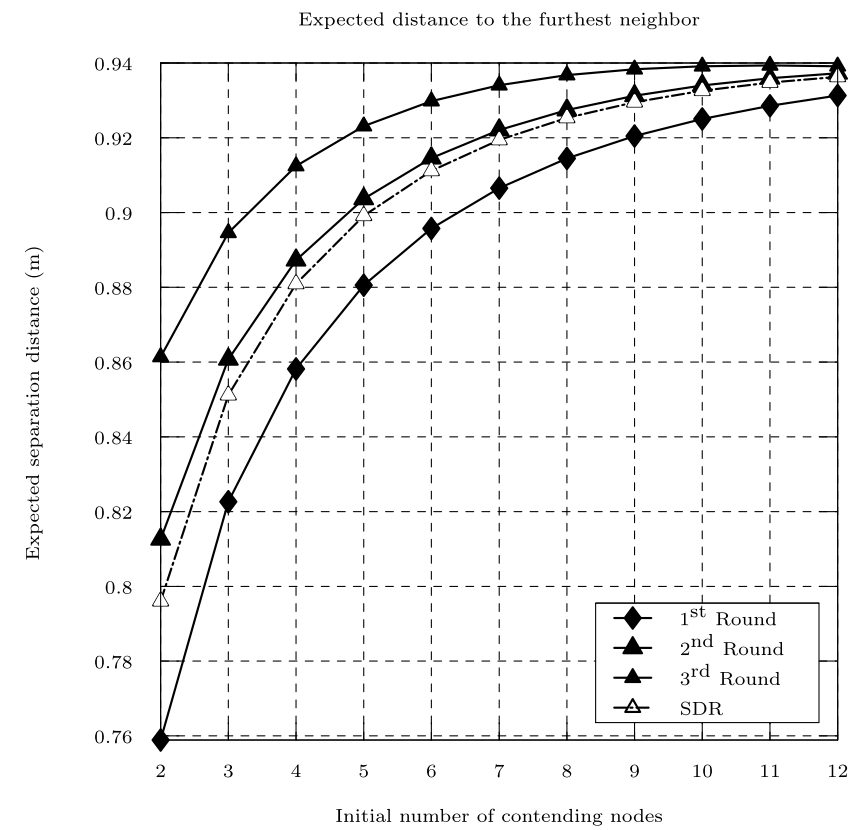

Fig. 11. Expected separation distance of the furthest neighbor for the geographical forwarding schemes. The source's transmission range is $R=1 \mathrm{~m}$ (normalized).

separation distance to the nearest and furthest eligible relays, respectively.

Both figures show the benefit of interactively using location information. Note that for the sectoral decision scheme, regardless of the previous outcome, the source node always readdresses the same original decision region for each of the subsequent rounds, while by employing the convex-lenses scheme, nodes make use of the outputs of previous rounds for future decisions. In this way, given that the decision regions closer to the destination enclose relays, the likelihood of hoping further increases each round.

In Fig. 10, the expected distance to the nearest relay reduces by increasing the density of nodes, but since the convex-lenses decision region is built towards the final destination and a cutoff boundary is intrinsically established, the region between source and this border is not searched for candidate relays (see Fig. 2). Therefore, the expected distance to the nearest neighbor increases at each iteration.

For the evaluated number of relays in Fig. 11, the distance to the furthest node does not increase substantially by considering more candidates. The more neighbors within the range, the greater is the expected distance to the furthest eligible relay.

Figs. 12 and 13 relate the expected distance to the $n$-th nearest eligible relay to the corresponding resolution interval length using the standard tree- and auction-based selection schemes. The numbers nearby the markers indicate the size of the initial set of colliding nodes. Regarding the expected advancement provided by the furthest neighbor strategy, both sectoral and convex-lenses schemes provide equivalent results. Conversely, the expected distance of the nearest node not only experience higher variance in hop length, but also the expected advancement become even smaller since the nearest node is found closer to the source when the density of candidate relays increases. 


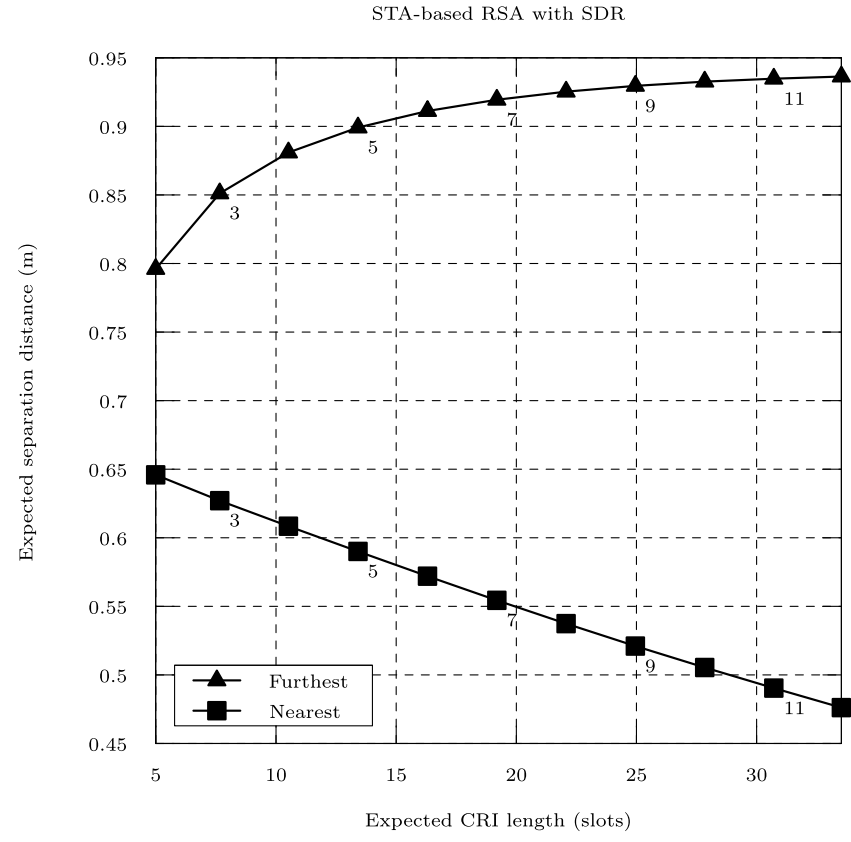

Fig. 12. Expected distance to the $n$th nearest neighbor related to the expected value of the length of the contention resolution interval for an increasing number of contending relays. The numbers nearby the markers designate the initial number of colliding nodes. The transmission range is $R=1 \mathrm{~m}$ (normalized).

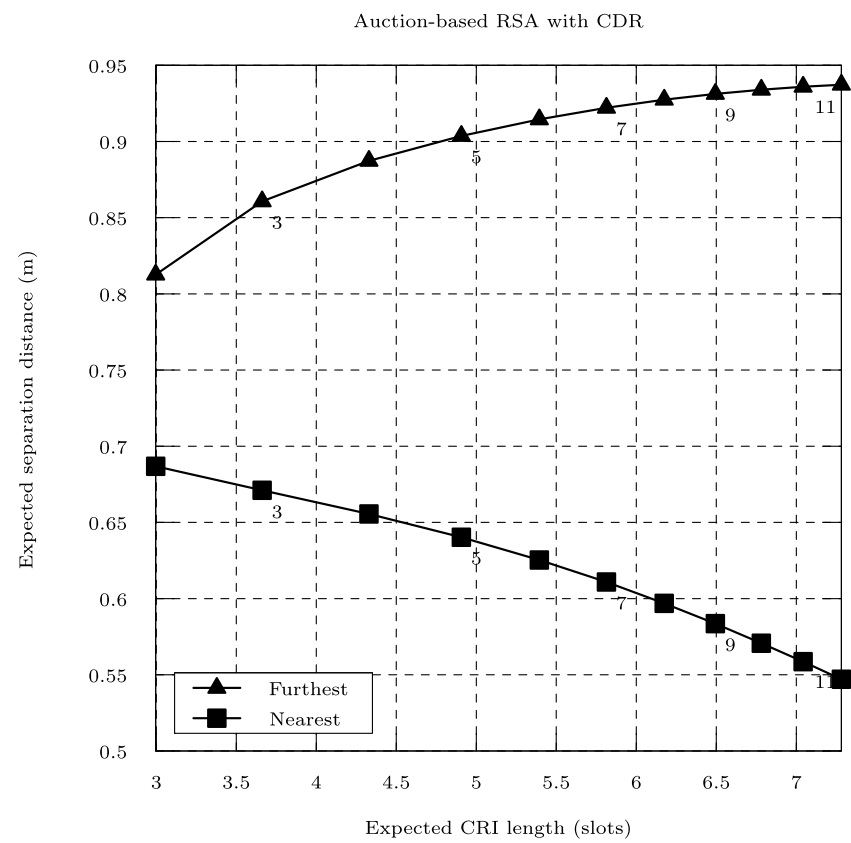

Fig. 13. Expected distance to the $n$th nearest neighbor related to the expected value of the length of the contention resolution interval for an increasing number of contending relays. The numbers nearby the markers designate the initial number of colliding nodes. The transmission range is $R=1 \mathrm{~m}$ (normalized).

From Figs. 12 and 13, the contention resolution interval works against the apparent benefit of having more eligible relays within the range. Depending on the initial number of colliding nodes, the contention resolution interactions may linger too long and then compromise the performance. It is then reasonable to maintain small the number of potential relays that get actively involved in the election process, because the selection procedure substantially contributes to the communication cost at hop-basis.

\section{Final REMARKS}

In this paper, we study contention-based geographic routing protocols by assessing their constituent operational parts, namely forwarding region design and relay selection algorithm. To do that, we establish an analytical framework based on stochastic geometry to measure the achievable progress at each hop, and on the probability generating function concept to obtain the distribution of time needed to select the most suitable relay. Using this framework, the length of the contention resolution interval required to find a relay in multihop scenarios is characterized and the progress that is enabled by the forwarding decision regions are described. Our results show that the proposed convex-lens auction-based approach using location information outperforms the random selection solution. The auction-based method substantially reduces the protocol overhead (relay selection) of establishing active connections in autonomous multi-hop networks allowing for more efficient reuse of the shared channel.

\section{REFERENCES}

[1] X. Liu, "Atypical hierarchical routing protocols for wireless sensor networks: A review," IEEE Sensors J., vol. 15, no. 10, pp. 5372-5383, Oct. 2015.

[2] K. Han, J. Luo, Y. Liu, and A. V. Vasilakos, "Algorithm design for data communications in duty-cycled wireless sensor networks: A survey," IEEE Commun. Mag., vol. 51, no. 7, pp. 107-113, Jul. 2013.

[3] I. Stojmenovic, "Machine-to-machine communications with in-network data aggregation, processing, and actuation for large-scale cyber-physical systems," IEEE Internet Things J., vol. 1, no. 2, pp. 122-128, Apr. 2014.

[4] F. Cadger, K. Curran, J. Santos, and S. Moffett, "A survey of geographical routing in wireless ad-hoc networks," IEEE Commun. Surveys Tuts., vol. 15, no. 2, pp. 621-653, May 2013.

[5] P. Stenumgaard, J. Chilo, P. Ferrer-Coll, and P. Angskog, "Challenges and conditions for wireless machine-to-machine communications in industrial environments," IEEE Commun. Mag., vol. 51, no. 6, pp. 187-192, Jun. 2013.

[6] F. Baccelli, B. Błaszczyszyn, and P. Mühlethaler, "Time-space opportunistic routing in wireless ad hoc networks: Algorithms and performance optimization by stochastic geometry," Comput. J., vol. 53, no. 5 , pp. 592-609, Jun. 2010.

[7] S. Srinivasa and M. Haenggi, "Combining stochastic geometry and statistical mechanics for the analysis and design of mesh networks," Ad Hoc Netw., vol. 13, pp. 110-122, Feb. 2014.

[8] P. H. J. Nardelli, P. Cardieri, and M. Latva-aho, "Efficiency of wireless networks under different hopping strategies," IEEE Trans. Wireless Commun., vol. 11, no. 1, pp. 15-20, Jan. 2012.

[9] D. Chen, J. Deng, and P. K. Varshney, "Selection of a forwarding area for contention-based geographic forwarding in wireless multi-hop networks," IEEE Trans. Veh. Technol., vol. 56, no. 5, pp. 3111-3122, Sep. 2007.

[10] C. H. M. de Lima and G. T. F. de Abreu, "Clusterization for robust geographic routing in wireless sensor networks," in Proc. IEEE WCNC, Mar./Apr. 2008, pp. 2385-2390.

[11] C. H. M. de Lima and G. T. F. de Abreu, "Analysis of contentionbased relay selection mechanisms in autonomous multi-hop networks," in Proc. IEEE ITW, Jun. 2009, pp. 186-190.

[12] C. H. M. de Lima and G. T. F. de Abreu, "Contention-based geographic forwarding strategies for position-centric routing," in Proc. IEEE 6th Workshop Positioning, Navigat. Commun. (WPNC), Mar. 2009, pp. 295-299.

[13] M. Haenggi, Stochastic Geometry for Wireless Networks. Cambridge, U.K.: Cambridge Univ. Press, 2012.

[14] H. ElSawy, E. Hossain, and M. Haenggi, "Stochastic geometry for modeling, analysis, and design of multi-tier and cognitive cellular wireless networks: A survey," IEEE Commun. Surveys Tuts., vol. 15, no. 3, pp. 996-1019, Jul. 2013. 
[15] D. Stoyan, W. S. Kendall, and J. Mecke, Stochastic Geometry and Its Applications, 2nd ed. New York, NY, USA: Wiley, 1995.

[16] A. Baddeley, I. Bárány, R. Schneider, and W. Weil, Stochastic Geometry. New York, NY, USA: Springer, 2006.

[17] S. Srinivasa and M. Haenggi, "Distance distributions in finite uniformly random networks: Theory and applications," IEEE Trans. Veh. Technol., vol. 59, no. 2, pp. 940-949, Feb. 2010.

[18] M. Zorzi, "A new contention-based MAC protocol for geographic forwarding in ad hoc and sensor networks," in Proc. IEEE Int. Conf. Commun., vol. 6, Jun. 2004, pp. 3481-3485.

[19] M. Franceschetti and R. Meester, Random Networks for Communication: From Statistical Physics to Information Systems. Cambridge, U.K. Cambridge Univ. Press, Jan. 2008.

[20] M. Haenggi, J. G. Andrews, F. Baccelli, O. Dousse, and M. Franceschetti, "Stochastic geometry and random graphs for the analysis and design of wireless networks," IEEE J. Sel. Areas Commun. vol. 27, no. 7, pp. 1029-1046, Sep. 2009.

[21] P. Mathys and P. Flajolet, " $Q$-ary collision resolution algorithms in random-access systems with free or blocked channel access," IEEE Trans. Inf. Theory, vol. 31, no. 2, pp. 217-243, Mar. 1985.

[22] S. Ruhrup, H. Kalosha, A. Nayak, and I. Stojmenović, "Messageefficient beaconless georouting with guaranteed delivery in wireless sensor, ad hoc, and actuator networks," IEEE/ACM Trans. Netw., vol. 18, no. 1, pp. 95-108, Feb. 2010.

[23] Y. Yu and G. B. Giannakis, "High-throughput random access using successive interference cancellation in a tree algorithm," IEEE Trans. Inf. Theory, vol. 53, no. 12, pp. 4628-4639, Dec. 2007.

[24] A. Papoulis and S. U. Pillai, Probability, Random Variables and Stochastic Processes, 4th ed. New York, NY, USA: McGraw-Hill, 2002.

[25] M. Abramowitz and I. A. Stegun, Handbook of Mathematical Functions: With Formulas, Graphs, and Mathematical Tables, 9th ed. New York, NY, USA: Dover, 1965.

[26] H. S. Wilf, Generatingfunctionology, 3rd ed. New York, NY, USA: Academic, 2005.

[27] J. Abate and W. Whitt, "Numerical inversion of probability generating functions," Oper. Res. Lett., vol. 12, pp. 245-251, Oct. 1992.

[28] S. Biswas and Y. Narahari, "Iterative Dutch combinatorial auctions," Ann. Math. Artif. Intell., vol. 44, no. 3, pp. 185-205, 2005.

[29] I. Amadou, A. Ouni, H. Rivano, and F. Valois, "Energy-efficient relay selection schemes for contention-based geographic forwarding," in Proc. IFIP Wireless Days, Nov. 2012, pp. 1-6.

[30] Z. Ji, W. Yu, and K. J. R. Liu, "A game theoretical framework for dynamic pricing-based routing in self-organized MANETs," IEEE J. Sel. Areas Commun., vol. 26, no. 7, pp. 1204-1217, Sep. 2008.

[31] K. P. Naveen and A. Kumar, "Relay selection for geographical forwarding in sleep-wake cycling wireless sensor networks," IEEE Trans. Mobile Comput., vol. 12, no. 3, pp. 475-488, Mar. 2013.

[32] H. Mohamed and P. Robert, "Dynamic tree algorithms," Ann. Appl. Probab., vol. 20, no. 1, pp. 26-51, 2010.

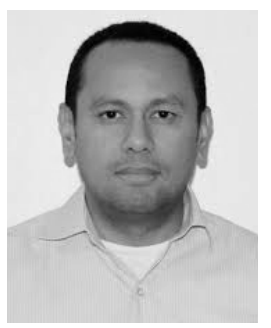

Carlos H. M. de Lima received the B.Sc. and M.Sc. degrees in electrical engineering from the Federal University of Ceará, Fortaleza, Brazil, in 2002 and 2004, respectively, and the D.Sc. degree in telecommunications engineering from the University of Oulu, Finland, in 2013. From 2000 to 2005, he was a Research Scientist with the Wireless Telecommunications Research Group, Fortaleza. In 2005, he was a Visiting Researcher with the Ericsson Research Center, Lulea, Sweden, engaged in power control techniques for enhanced high-speed packet access systems. In 2006, he was with the Nokia Institute of Technology, Brazil, engaged in the evaluation of the system performance of WiMAX systems. He is currently an Assistant Professor with São Paulo State University, São João da Boa Vista-SP, Brazil, and a member of the Research Staff with the Centre for Wireless Communications, University of Oulu. His research interests include statistical signal processing and analysis of interference networks using stochastic geometry

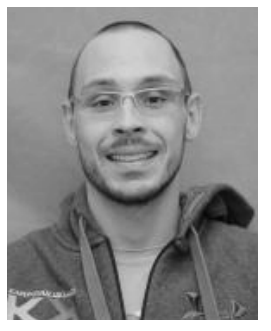

Pedro Henrique Juliano Nardelli received the B.S. and M.Sc. degrees in electrical engineering from the State University of Campinas, Brazil, in 2006 and 2008, respectively, and the Doctoral degree from the University of Oulu, Finland, and the State University of Campinas, in 2013, following a dualdegree agreement. He currently holds a post-doctoral position at the University of Oulu, and his studies are mainly focused on the efficiency of wireless networks and spatiotemporal dynamics of complex systems.

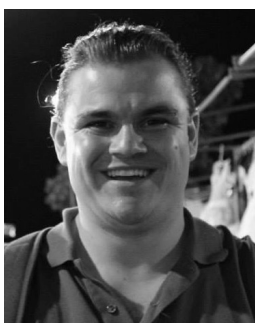

Hirley Alves received the B.Sc. and M.Sc. degrees in electronic engineering from the Federal University of Technology-Paraná, Brazil, in 2010 and 2011, respectively. He has participated in the Capes/Brafitec undergraduate exchange program, spending one year at the Universite de Technologie de Troyes, France. He is currently enrolled in a dual degree Ph.D. program between the University of Oulu and the Federal University of TechnologyParaná. He has been a Research Assistant with the Centre for Wireless Communications, University of Oulu, Oulu, Finland. His research interests lie in: wireless and cooperative communications; coding and HARQ techniques; full-duplexing communications; PHY-security networks; and performance analysis of practical fullduplex relaying schemes, and its application on heterogeneous networks.

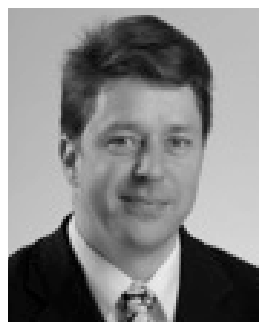

Matti Latva-aho was born in Kuivaniemi, Finland, in 1968. He received the M.Sc., Lic.Tech., and D.Tech. (Hons.) degrees from the University of Oulu, Finland, in 1992, 1996, and 1998, respectively, all in electrical engineering. From 1992 to 1993, he was a Research Engineer with Nokia Mobile Phones, Oulu. From 1994 to 1998, he was a Research Scientist with the Telecommunication Laboratory and the Centre for Wireless Communications at the University of Oulu. He was the Director of the Centre for Wireless Communications with the University of Oulu from 1998 to 2006. $\mathrm{He}$ is currently the Department Chair Professor of Digital Transmission Techniques and Head of the Department for Communications Engineering, University of Oulu. He has authored over 200 conference or journal papers in the field of wireless communications. His research interests are related to mobile broadband wireless communication systems. He has been the TPC Chairman for PIMRC06, the TPC Co-Chairman for ChinaCom07, and the General Chairman for WPMC08. He acted as the Chairman and the Vice Chairman of the IEEE Communications Finland Chapter from 2000 to 2003. 